\title{
Limb Salvage for Primary Malignant Bone Tumors of the Distal Leg in Skeletally Immature Patients: Ankle Arthrodesis Using Vascularized Ipsilateral or Contralateral Fibular Flap
}

\author{
Ahmed Mohamed El Ghoneimy, MD ${ }^{1}$ Mohamed Sokar, MSc ${ }^{1} \quad$ Nehal Kamal, BSc ${ }^{1}$ \\ ${ }^{1}$ Department of Orthopedic, Children Cancer Hospital Egypt, Cairo, \\ Egypt \\ Address for correspondence Ahmed Mohamed El Ghoneimy, MD, \\ Department of Orthopedic, Children Cancer Hospital Egypt, Cairo \\ J Reconstr Microsurg Open 2019;4:e36-e41. \\ University, 1 Seket El Emam, Sayeda Zeinab, Cairo, 26386, Egypt \\ (e-mail: ahmed.elghoneimy@57357.org).
}

\begin{abstract}
Background Malignant bone tumors of the distal tibia or fibula in children are rare. Quality of functional outcome following limb salvage surgery is still controversial. This is a retrospective review of the functional outcome of ankle arthrodesis using vascularized fibular flap.

Methods A total of seven patients were reviewed. The diagnosis was osteosarcoma in five and Ewing's sarcoma in two patients. The primary tumor site was the distal tibia in six patients and the distal fibula in one patient. The average age at presentation was 10.6 years (range, 6.7-14 years). The average follow-up period was 24.5 months (range, 13-69 years). A pedicled fibular graft was harvested from the ipsilateral leg in two patients and a free vascularized osteocutaneous fibular flap from the contralateral leg in five patients. External fixation was used in five and internal fixation in two patients. Patients were allowed full weight bearing following radiographic evidence of complete bone union.

Results The mean time to complete bone union and full weight bearing was 7.1 months (range, 4-13 months). One patient developed nonunion at graft-host junction and one patient had stress fracture of the fibular graft. The mean limb length

\section{Keywords}

- osteosarcoma

- Ewing's sarcoma

- distal tibia

- vascularized fibular graft

- ankle arthrodesis inequality was $0.57 \mathrm{~cm}$ (range, $0-3 \mathrm{~cm}$ ). The average Musculoskeletal Tumor Society (MSTS) score was $84.5 \%$ (range, $73-100 \%$ ).

Conclusion Skeletally immature patients treated by ankle arthrodesis using vascularized fibular flap can return to full weight bearing within the first year following surgery. A contralateral fibular flap has the advantage of preserving the contour of the ankle and reserving the ipsilateral fibula for initial stability at the fusion site. This study is of level IV evidence.
\end{abstract}

Advances in neoadjuvant chemotherapy and surgical reconstruction techniques rendered limb preservation possible in most malignant bone tumor patients. ${ }^{1}$ However, limb salvage for malignant tumors around the ankle joint is still

\footnotetext{
DAhmed Mohamed El Ghoneimy's ORCID is https://orcid.org/ 0000-0003-2105-8749.
}

received

December 11, 2018 accepted after revision March 18, 2019
DOI https://doi.org/

$10.1055 / \mathrm{s}-0039-1688762$. ISSN 2377-0813. associated with higher incidence of complications, as compared with amputation due to subcutaneous location of the distal tibia and fibula, tendinous nature of the surrounding musculature, and proximity of neurovascular structures. These factors lead to limitations in local soft tissue coverage and to the difficulty to obtain safe surgical margins. ${ }^{2}$ Another challenging problem is the relatively young age of patients
Copyright $\odot 2019$ by Thieme Medical Publishers, Inc., 333 Seventh Avenue, New York, NY 10001, USA. Tel: +1(212) 584-4662.
License terms

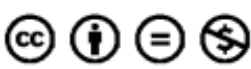


diagnosed with primary malignant bone tumors, the majority of which are skeletally immature, with high functional demands and a remaining growth potential. ${ }^{1}$

Reports on intra-articular resections of malignant bone tumors of the distal tibia and fibula in children and adolescents are limited and often comprise small number of patients due to the low incidence of primary malignant bone tumors in this anatomic location. ${ }^{3}$ Reconstructive options include ankle arthrodesis using bone autografts or allografts, or a mobile ankle reconstruction using endoprosthesis, each having its advantages and disadvantages. ${ }^{4-6}$

The aim of this work is to retrospectively evaluate the surgical technique and functional outcome of ankle arthrodesis using a vascularized fibular autograft in skeletally immature bone tumor patients.

\section{Methods}

All patients diagnosed with a malignant bone tumor of the distal tibia or fibula in the interval between 2008 and 2017 were reviewed. The inclusion criteria were patients for whom an intra-articular resection of the distal tibia and/or fibula and reconstruction of the resulting bone defect was done by ankle arthrodesis using a vascularized fibular bone graft. The exclusion criteria were: (1) tumors of the distal tibia or fibula, not reaching the articular surface, and for which an ankle joint sparing resection was done. These were considered as intercalary tibial or fibular bone resections that should be evaluated separate from the current analysis. (2) Tumors of the distal fibula for which an intra-articular resection was done without reconstruction.(3)Patients with locally advanced tumor necessitating a below knee amputation. (4) Patients with a follow-up period of less than 12 months since limb salvage surgery.

A total of seven patients met the inclusion criteria. The mean age at presentation was 10.6years (range, 6.7-14 years). There were three male and four female patients. The diagnosis was osteosarcoma in five patients and Ewing's sarcoma in two patients. All patients received neoadjuvant and adjuvant chemotherapy. None of the patients received radiotherapy neither preoperative nor postoperative. Planning for limb salvage was based on the evaluation of the preoperative magnetic resonance imaging (MRI) for safe bone and soft tissue resection margins. Proximal resection margins were planned at $2 \mathrm{~cm}$ away from the intramedullary extent of the tumor, while distally, the articular cartilage of the distal tibia and/or fibula were considered as a distal safe margin. The mean length of bone defect after resection was $13.2 \mathrm{~cm}$ (range, 8-24 cm). In one patient, diagnosed with Ewing's sarcoma of the distal fibula, the soft tissue extent of the tumor was reaching the lateral cortex of the adjacent distal tibia and an enblock resection of the distal fibula and hemi cortex of the tibia was done.

Reconstruction was done in all patients by ankle arthrodesis using a fibular flap. Reconstruction with ipsilateral pedicled vascularized fibular flap (centralization of the ipsilateral fibula): This method of reconstruction was first described by Ebeid et al. ${ }^{7}$ We applied this technique in the first two patients diagnosed with a distal tibia osteosarcoma.
Through the same anterior midline approach used for the resection, the ipsilateral fibula was dissected from the attached muscles. The peroneal vessels were proximally dissected and mobilized with the fibular graft following proximal osteotomy and distally the vessels were ligated to be able to mobilize and trough the graft into talar dome after the disarticulation of the lateral malleolus from the talofibular joint. The advantage of this technique compared with using a contralateral free vascularized fibular graft is that it does not require microvascular reconstruction, and it avoids the donor site morbidity in the contralateral leg. ${ }^{7}$ Reconstruction with contralateral vascularized osteocutaneous free fibular flap: In the following five patients, we changed the method of reconstruction from an ipsilateral fibular graft to a contralateral free vascularized osteocutaneous fibular flap. The reasons for changing the method of reconstruction were to preserve the ipsilateral fibula and consequently facilitate proper alignment of the limb during fixation and to provide a skin island with the bone graft to facilitate the soft tissue closure and coverage of the defect. The average skin flap size was 7 and $3.5 \mathrm{~cm}$ in craniocaudal and transverse dimensions, respectively. As in the previously described technique of the pedicled fibular graft, the free fibular graft was troughed proximally into medulla of the tibia and distally into talar dome. Proper adjustment of the rotation of the graft was done in a manner so that the peroneal pedicle comes to lie in line with the anterior tibial vessels (donor vessels). Finally, the anastomosis of the peroneal vessels to the anterior tibial vessels was performed in an end-to-end fashion and the skin island was used for closure of the anterior skin. At the donor site, the skin defect at site of previously harvested skin island was left to heal by secondary intention.

Internal fixation was used in two patients (a 3.5 reconstruction plate with proximal screws fixed to the tibial shaft and distal screws fixed to talus). An external ring fixator was applied in the other five patients with combination of shanz screws and tensioned wires in the tibial shaft proximal to implanted fibula and distally into the talus and calcaneus. All patients remained nonweight bearing until periosteal new bone formation was radiographically observed at the proximal and distal ends of the fibular graft, after which, partial weightbearing was allowed using two crutches and a below knee cast. Full weight bearing without support or cast was allowed following the radiographic evidence of complete bone union and graft hypertrophy (-Fig. 1).

Follow-up was at 6 weekly intervals in the first 6 months, 3 monthlies in the following 2 years, and 6 monthlies thereafter. Functional evaluation was done using the musculoskeletal tumor society scoring system ${ }^{8}$ and radiographic evaluation was done using anteroposterior and lateral radiographs. To check for local recurrence, a local MRI was done at 6 months interval, in the first 5 years following surgery.

\section{Results}

The mean follow-up period was 24.5 months (range, 1369 months). 

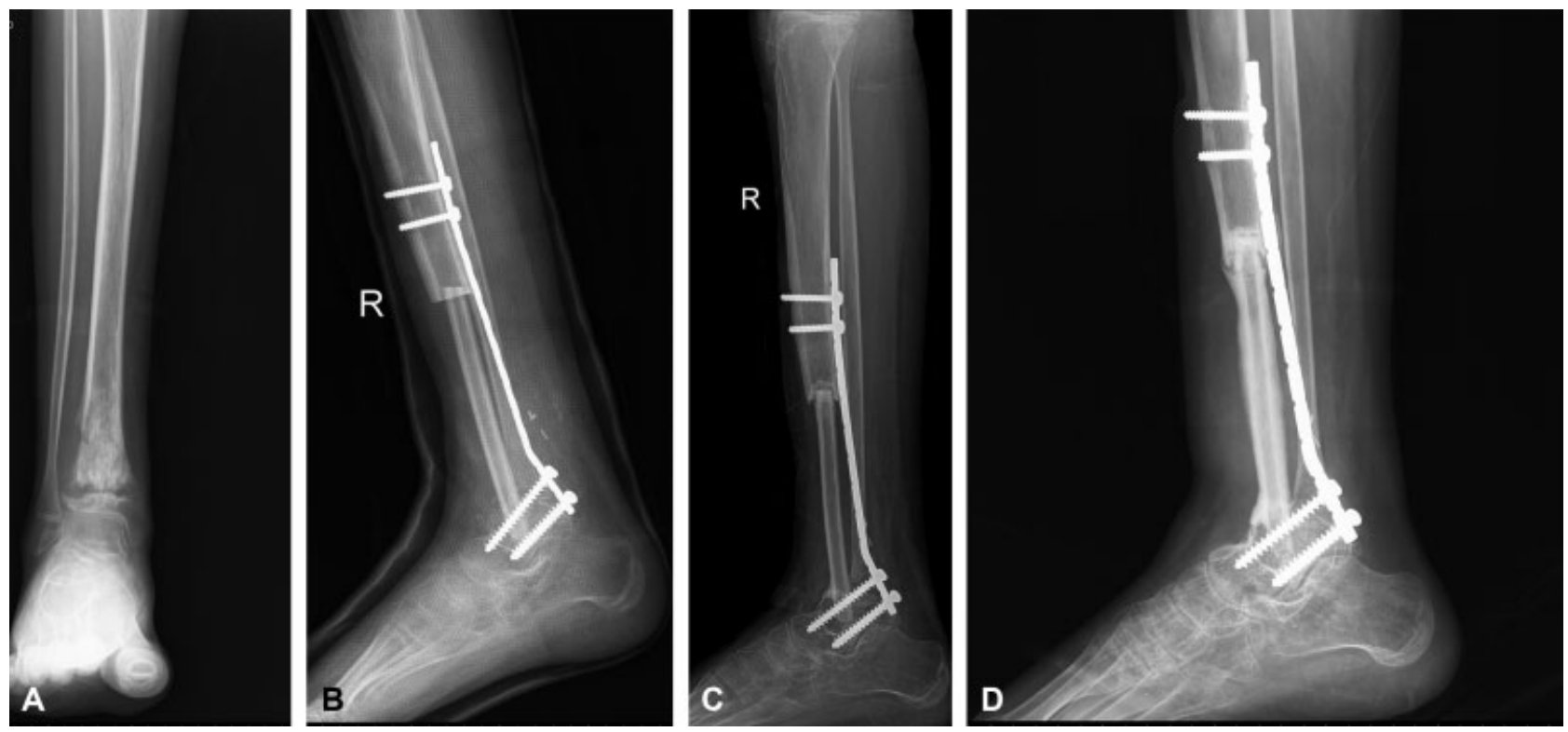

Fig. 1 (A) Preoperative anteroposterior radiograph of an osteosarcoma involving the distal tibia in a 12-year-old male patient. (B) Postoperative lateral radiograph following the resection of the distal tibia and ankle arthrodesis using a contralateral vascularized fibular graft and fixation by a 4.5 reconstruction plate. Limb is immobilized in a below knee cast. (C) Lateral radiograph 3 months postoperative showing periosteal new bone formation at the proximal and distal junction of the fibular graft. (D) Lateral radiograph 9 months postoperative showing hypertrophy of the graft.

Oncologic outcome: At diagnosis, three patients were nonmetastatic and four (two osteosarcoma and two Ewing's sarcoma) were initially metastatic to lung. Surgical margins were negative in all patients. All patients, with exception of one, in which tumor necrosis could not be retrieved from medical records, had good response to chemotherapy with a tumor necrosis above $90 \%$. No patient had evidence of local relapse during their follow-up period. Two patients, diagnosed with Ewing's sarcoma and lung metastases at presentation, had complete resolution of their lung metastases during their neoadjuvant chemotherapy and had no evidence of further lung metastases until their latest follow-up visit. Two patients diagnosed with osteosarcoma of the distal tibia and initial lung metastases had metastasectomy following limb salvage surgery. One patient remained free from disease until his latest follow-up and the other patient developed subsequent inoperable lung metastases and died from disease 22 months later. Out of the seven patients, six remained with no evidence of disease until their latest follow-up period.

Bone union and graft hypertrophy: The mean time for radiographic evidence of periosteal new bone formation at the proximal and distal graft-host junction was observed at 2.6 months (range, 2.3-6.5 months). Consolidation of bone union and partial graft hypertrophy was observed following a mean time of 7.1 months (range, 4-13 months).

Functional outcome: Partial weight bearing in a below knee cast started at a mean duration of 3 months (range, 2.37 months). Full weight bearing, without cast, and without support started at a mean duration of 7.1 months (range, 413 months), as calculated from the date of surgery. The mean Musculoskeletal Tumor Society (MSTS) functional score ${ }^{8}$ was $84.5 \%$ (range, $73-100 \%$ ). All patients had occasional pain, not requiring medication, were able to walk unsupported with a minor limp, and had returned to their school activity within the 1 st year following surgery ( - Video $\mathbf{1}$ ).

\section{Video 1}

Clinical outcome of a 9-year-old female patient 2 years following surgery. Online content including video sequences viewable at: https://www.thiemeconnect.com/products/ejournals/html/10.1055/ s-0039-1688762.

Complications: Nonunion occurred in one patient. This patient was diagnosed with an osteosarcoma of the distal tibia and was treated by intra-articular resection and ankle arthrodesis using an ipsilateral pedicled fibular graft. Nonunion developed at the proximal graft-host junction, 9 months following the primary surgery. Cast was removed, external fixation was reapplied and autologous iliac crest bone graft was added to nonunion site. Bone healing with a residual varus angulation at the junction site was evident 3 months later ( - Fig. 2). Patient has been walking since unsupported with no pain and a mild limp, refusing to do any corrective surgery for the varus deformity. A wound dehiscence developed at the junction of the cutaneous flap and the original skin incision in one patient 3 days following surgery and was managed by secondary sutures with eventual healing. One patient developed a stress fracture of his fibular graft 7 months following surgery. The patient was restricted from weight bearing and complete healing of the fracture was observed 3 months later (-Fig. 3). A mean limb length discrepancy of $0.57 \mathrm{~cm}$ (range, $0-3 \mathrm{~cm}$ ) was measured at the latest clinical evaluation. 

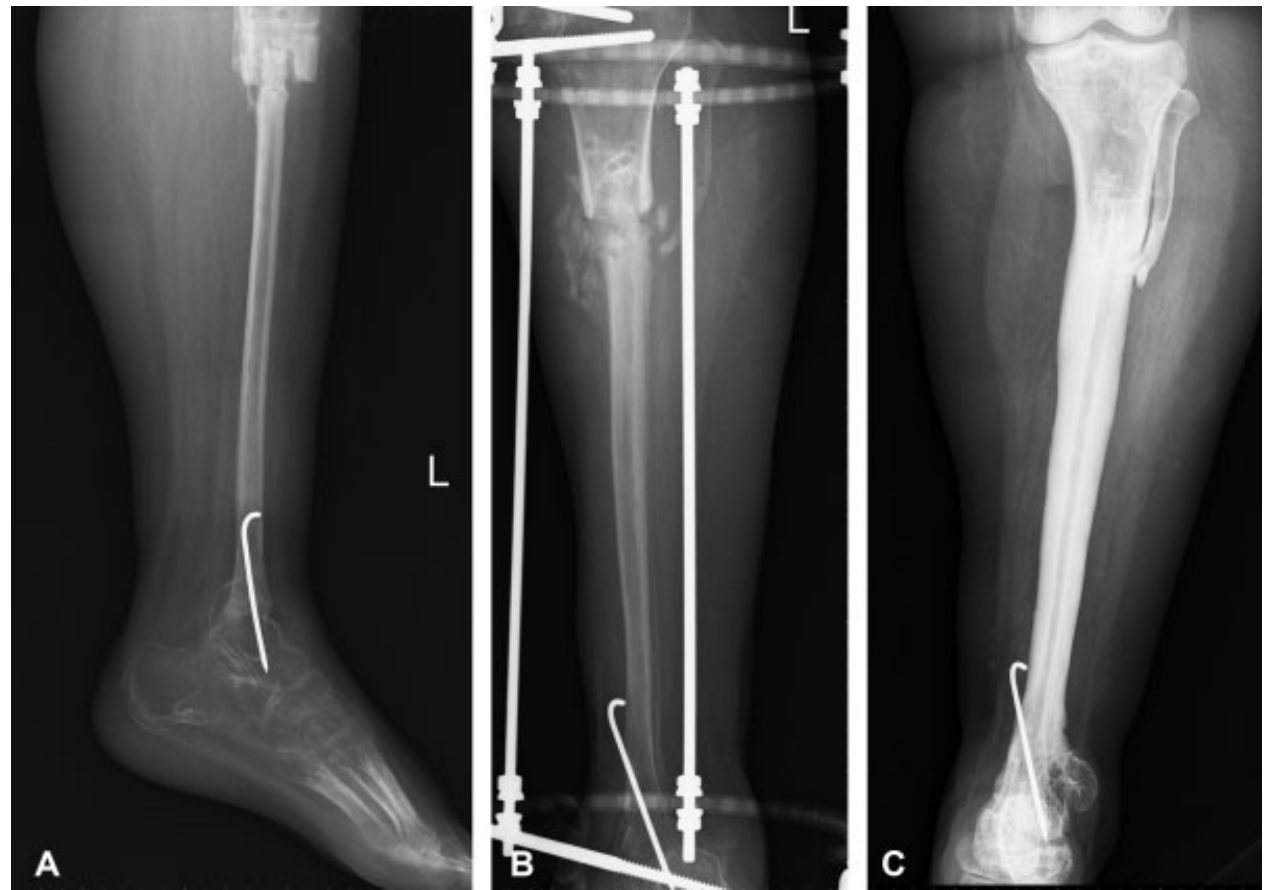

Fig. 2 (A) A 14-year-old female patient diagnosed with an osteosarcoma of the distal tibia. Lateral radiograph of the leg, 9 months postoperative, showing proximal nonunion at the junction between tibia and an ipsilateral pedicled fibular graft (centralization of the fibula). (B) Anteroposterior radiograph following revision of fixation using ring fixator and iliac crest bone graft. (C) Anteroposterior radiograph 36 months following surgery showing complete union and hypertrophy of the graft.
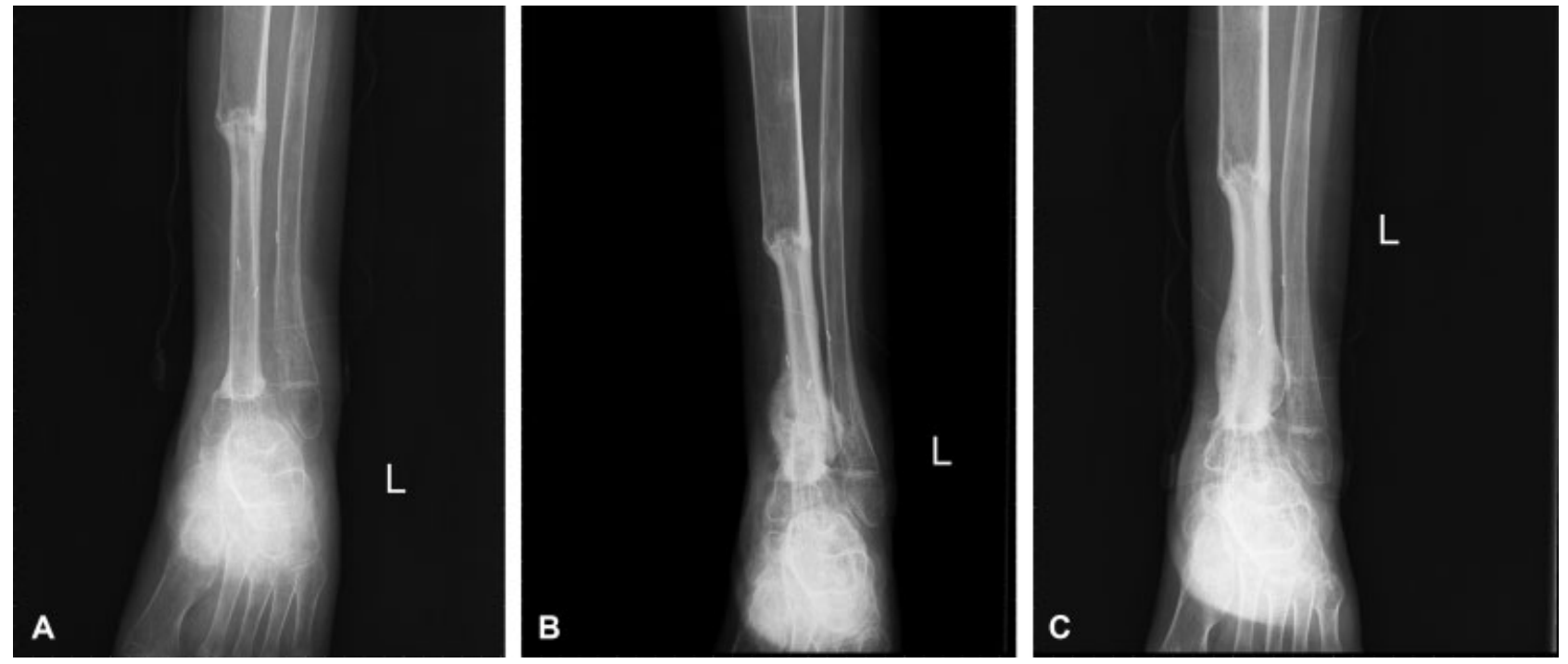

Fig. 3 (A) A 13-year-old male diagnosed with an osteosarcoma of the distal tibia. Anteroposterior radiograph of the distal leg, 4 months postoperative, showing complete proximal and distal union of a contralateral vascularized fibular graft. (B) Anteroposterior radiograph showing hypertrophy and a stress fracture in the fibular graft 7 months postoperative with abundant callus surrounding the fracture. (C) Anteroposterior radiograph 3 months following fracture showing healing and complete remodeling of the surrounding callus.

\section{Discussion}

Mavrogenis et $\mathrm{al}^{2}$ published the largest series on osteosarcoma of the distal tibia in 42 patients. They compared amputation with limb salvage and found that, although there was higher incidence of local recurrence and complications in the limb salvage group compared with amputation, the survival in both groups was the same but function was superior in limb salvage group. They concluded that it may be worthwhile to accept these higher risks, with strict follow-up of selected patients for whom limb salvage is feasible and reserve amputation for patients with local recurrence. Tillman ${ }^{9}$ stated that in the practice of his institute, they advise patients that their function, in the long term, is likely to be inferior with limb salvage surgery as opposed to expected function from a modern below knee prosthesis. Most of literature on tumors around the ankle comprises level IV evidence (case series) due to the low frequency of malignant bone tumors in this area. Accordingly, 
there is no clear consensus on the oncologic and functional benefit of limb salvage versus amputation in this anatomic location. In the authors' institute, we explain to patients the higher risk of complications and possibility of multiple revision surgeries associated with limb salvage surgery as compared with amputation.

Reconstruction of the ankle joint using custom made megaprosthesis has been previously reported with a good functional outcome. ${ }^{6,10,11}$ Yang et al published the largest series and they had complications in $37 \%$ of their patients including deep infection and loosening of the talar component. ${ }^{6}$ Despite the disadvantage of the loss of ankle motion following ankle arthrodesis as compared with a mobile ankle reconstruction using endoprosthesis, we preferred ankle arthrodesis using vascularized fibular flap in our group of patients due to the following factors: (1) a great majority of patients diagnosed with osteosarcoma or Ewing's sarcoma are children or adolescents with high functional demand and relatively longer life expectancy. A long-lasting biologic reconstruction would be more suitable for this age group. (2) Most patients are diagnosed with malignant bone tumors and receive chemotherapy with a higher risk for wound infection, especially for reconstructions around the ankle with limited soft tissue coverage. Vascularized fibular flaps are less bulky, more resistant to infection owing to their vascularity, and provide additional soft tissue coverage when harvested with a skin island. (3) All reported series on megaprosthetic reconstructions of the ankle describe the use of custom-made implants with a minimum of 6 weeks for delivery of the implant and a high cost.

Various types of bone grafts have been used for ankle arthrodesis. These include intercalary allografts, ${ }^{5,12,13}$ contralateral autologous tibial graft, ${ }^{5,14}$ pedicled ipsilateral autologous fibular flap, ${ }^{7,15,16}$ and free vascularized contralateral autologous fibular flap. ${ }^{14}$ The advantages and disadvantages of allograft and autografts are well documented in literature. ${ }^{17,18}$ Size-matched allografts provide initial strength and stability for the construct, while vascularized flaps provide a faster and higher rate of bone healing and progressive increase in strength as the graft hypertrophies. ${ }^{19,20}$ The Capanna's technique, combining allograft and vascularized fibula, has been described for reconstruction of several long-bone defects but, to our knowledge, it has not been used for ankle arthrodesis. ${ }^{21}$ This may be related to the fact that the reconstruction is too bulky for the ankle as suggested by Scaglioni et al. ${ }^{14}$ Moore et al published their results on ankle arthrodesis using allograft in nine tumor patients. ${ }^{12}$ Six of nine patients required additional surgery including two cases of graft fracture that were managed by iliac crest bone grafting and revision of fixation. Comparing our results, we had one case of stress fracture of the fibular graft which healed conservatively without the need for a revision surgery. Fractures of large segment structural bone grafts are major concern in musculoskeletal reconstructions and vascularized grafts have the advantage over allografts for the ability to heal spontaneously. ${ }^{19}$

Several authors preferred the technique of centralization of the ipsilateral fibular graft into tibial defect ${ }^{7,15,16}$ while Scaglioni et $\mathrm{al}^{14}$ published their results on the use of contralateral free vascularized fibular flap. Although we used both techni- ques, the small number of patients in both groups does not allow for a statistical comparison of the outcome. However, a better cosmetic appearance (preserved contour) of the ankle was evident in patients treated by contralateral free osteocutaneous fibular flap and this may be attributed to the preserved ipsilateral lateral malleolus and the skin flap used for wound closure. Preserving the ipsilateral fibula also act as a guide for proper alignment of the limb during the implantation of the free fibula. This was in accordance with the series reported by Scaglioni et al. ${ }^{14}$ They used the same technique in five patients. The main differences from current study were: the average age of their patients which was 33.2 years compared with 10.6 in the current analyses, and the two-stage technique they used whereby they implanted the free fibular flap in a second surgery following a primary resection and spacer implantation. Their argument for this technique was the need to assess the surgical safety margins and avoid delay in chemotherapy after primary resection. We did not find delaying reconstruction to a second surgery necessary in our patients, as we assessed marrow margins intraoperatively using frozen section pathology and none of our patients had wound problems that would have delayed the postoperative chemotherapy.

The type of fixation used for ankle fusion varied in literature, between external as described by Shalaby et al ${ }^{16}$ and minimal internal fixation and cast as described by Ebeid et al. $^{7}$ We found that external fixation offers a more rigid compression of the fibular graft at the proximal and distal osteotomy sites and facilitates the postoperative wound care as compared with internal fixation and casting.

Limb length discrepancy is a major concern in resections of malignant bone tumors around the knee in skeletally immature patients. ${ }^{1}$ In distal tibial tumors, due to the limited share in growth by distal tibial physis, the expected limb length difference is usually minimal, as shown by Stéphane et al. ${ }^{22}$ Although a mean inequality of less than $1 \mathrm{~cm}$ was recorded in our patients, one of the limitations in the current study is the relatively short follow-up period. A longer follow-up study is needed for assessment of the final limb length difference at skeletal maturity.

\section{Limitations}

The major limitation in the current analysis was the small number of patients. Other case series on resections of distal tibial tumors had similar limitations. This makes a statistically representative comparison between various types of reconstruction difficult. A prospective multicenter cooperative study is strongly needed.

\section{Conclusion}

In conclusion, ankle arthrodesis using a vascularized fibular flap provides a rigid reconstruction of the ankle joint area and young patients are able to return to their daily life activity within the first year following surgery. The use of the contralateral osteocutaneous fibular flap provides soft tissue coverage and better shape of the ankle joint as compared with ipsilateral fibular graft. 


\section{Compliance with Ethical Standards}

1. All contributing authors have no conflict of interest to declare and no source of funding for the current project.

2. No research was done on human or animal subjects.

3. Scientific committee approval was obtained before conducting current retrospective analyses.

4. All patients signed an informed consent before any surgery.

The authors received no financial support for the research, authorship, and/or publication of this article.

\section{Funding}

None.

\section{Conflict of Interest}

None declared.

\section{References}

1 Most MJ, Sim FH. Limb salvage in skeletally immature patients with extremity sarcoma. In: San-Julian M, ed. Canadell's Pediatric Bone Sarcomas: Epiphysiolysis before Excision. 2nd ed. New York, NY: Springer Cham; 2016:75-101

2 Mavrogenis AF, Abati CN, Romagnoli C, Ruggieri P. Similar survival but better function for patients after limb salvage versus amputation for distal tibia osteosarcoma. Clin Orthop Relat Res 2012;470 (06):1735-1748

3 Chou LB, Ho YY, Malawer MM. Tumors of the foot and ankle: experience with 153 cases. Foot Ankle Int 2009;30(09):836-841

4 Kundu ZS, Gogna P, Gupta V, et al. Ankle fusion with centralisation of the fibula after distal tibia bone tumour resection. J Orthop Traumatol 2014;15(02):95-101

5 Campanacci DA, Scoccianti G, Beltrami G, Mugnaini M, Capanna R. Ankle arthrodesis with bone graft after distal tibia resection for bone tumors. Foot Ankle Int 2008;29(10):1031-1037

6 Yang P, Evans S, Khan Z, Abudu A, Jeys L, Grimer R. Reconstruction of the distal tibia following resection of aggressive bone tumours using a custom-made megaprosthesis. J Orthop 2017;14(03):406-409

7 Ebeid W, Amin S, Abdelmegid A, Refaat Y, Ghoneimy A. Reconstruction of distal tibial defects following resection of malignant tumours by pedicled vascularised fibular grafts. Acta Orthop Belg 2007;73(03):354-359

8 Enneking WF, Dunham W, Gebhardt MC, Malawar M, Pritchard DJ. A system for the functional evaluation of reconstructive procedures after surgical treatment of tumors of the musculoskeletal system. Clin Orthop Relat Res 1993;(286):241-246
9 Tillman RM. Prosthetic replacement for tumor of the distal tibia. In: Sim FH, Choong PFM, Weber KL, eds. Master Techniques in Orthopedic Surgery: Orthopaedic Oncology and Complex Reconstruction. Philadelphia, PA: Lippincott Williams \& Wilkins; 2011: 277-281

10 Natarajan MV, Annamalai K, Williams S, Selvaraj R, Rajagopal TS. Limb salvage in distal tibial osteosarcoma using a custom mega prosthesis. Int Orthop 2000;24(05):282-284

11 Shekkeris AS, Hanna SA, Sewell MD, et al. Endoprosthetic reconstruction of the distal tibia and ankle joint after resection of primary bone tumours. J Bone Joint Surg Br 2009;91(10):1378-1382

12 Moore DR, Halpern JL, Schwartz HS. Allograft ankle arthrodesis: a limb salvage technique for distal tibial tumors. Clin Orthop Relat Res 2005;440(440):213-221

13 Xu L, Zhou J, Wang Z, Xiong J, Qiu Y, Wang S. Reconstruction of bone defect with allograft and retrograde intramedullary nail for distal tibia osteosarcoma. Foot Ankle Surg 2018;24(02):149-153

14 Scaglioni MF, Arzi RY, Gur E, et al. Free fibula reconstruction of distal tibial defects after sarcoma surgery. Ann Plast Surg 2015;74 (06):680-683

15 Puri A, Subin BS, Agarwal MG. Fibular centralisation for the reconstruction of defects of the tibial diaphysis and distal metaphysis after excision of bone tumours. J Bone Joint Surg Br 2009; 91(02):234-239

16 Shalaby S, Shalaby H, Bassiony A. Limb salvage for osteosarcoma of the distal tibia with resection arthrodesis, autogenous fibular graft and Ilizarov external fixator. J Bone Joint Surg Br 2006;88 (12):1642-1646

17 Aponte-Tinao LA, Ayerza MA, Muscolo DL, Farfalli GL. Should fractures in massive intercalary bone allografts of the lower limb be treated with ORIF or with a new allograft? Clin Orthop Relat Res 2015;473(03):805-811

18 de Boer HH, Wood MB, Hermans J. Reconstruction of large skeletal defects by vascularized fibula transfer. Factors that influenced the outcome of union in 62 cases. Int Orthop 1990;14(02):121-128

19 El Ghoneimy A, El Sherbiny M, Kamal N. Use of vascularized fibular free flap in the reconstruction of the femur in pediatric and adolescent bone sarcomas: complications and functional outcome. J Reconstr Microsurg 2019;35(02):156-162

20 Estrella EP, Wang EH. A comparison of vascularized free fibular flaps with nonvascularized fibular grafts for reconstruction of long bone defects after tumor resection. J Reconstr Microsurg 2017;33(03):194-205

21 Capanna R, Bufalini C, Campanacci M. A new technique for reconstruction of large metadiaphyseal bone defects; a combined graft (allograft shell plus vascularized fibula). Orthop Traumatol 1993;2(03):159-177

22 Stéphane S, Eric M, Philippe W, Félix DJ, Raphael S. Resection arthrodesis of the ankle for aggressive tumors of the distal tibia in children. J Pediatr Orthop 2009;29(07):811-816 